\title{
Alessandro Ciallella \\ Research perspective on multiphysics and multiscale materials: a paradigmatic case
}

Published online: 15 May 2020

(C) Springer-Verlag GmbH Germany, part of Springer Nature 2020

\begin{abstract}
Pantographic fabrics are presented as a paradigmatic example to discuss the research perspective on multiphysics and multiscale materials. Reduced-order modeling, obtained by introducing higher-gradient or microstructured continua, shows much smaller computational needs than those required by full-scale 3D modeling calculations and by equivalent discrete spring systems. Researches already available in the literature compare theoretical predictions with results obtained in real experiments, analyzing both in-plane and outof-plane deformations possibly induced by local buckling phenomena. The goal is to achieve three major objectives: (1) formulate coarse-scale nonlinear higher-gradient continuum models describing a more general class of pantographic metamaterials, based on finer-scale descriptions, through homogenization techniques; (2) implement finite element analyses with shape functions possessing higher regularity or employing mixed formulations to perform simulations with the above-formulated models; and (3) validate and verify the derived models through the acquisition and analysis of experimental data. These goals would likely push toward the improvement of 3D printing protocols to enhance the quality of the pantographic prototypes and DIC as experiments measurement technique. It can be also conjectured that many macroscopic deformation energies can be synthesized by using as elementary elements, inside periodicity cells, some pantographic modules.
\end{abstract}

Keywords Pantographic fabrics $\cdot$ Multiphysics $\cdot$ Multiscale metamaterials $\cdot$ Higher-order gradient continuum models $\cdot$ Homogenization

\section{Introduction}

In the last years, the progress in the technical capability of designing and building materials at small scales (e.g., 3D printing, electrospinning, roll-to-roll processing, dry etching, wet chemical etching, wet bulk micromachining, photolithography and next-generation lithography, micromolding and self-assembly) have led to the realization of newly conceived materials, "metamaterials," whose peculiar mechanical and acoustical macroscopic (or coarse scale, i.e., at a length scale much larger than that of the basic unit cell) properties appear to have no equals in nature [10]. Often, especially in the elastic regimes, it is the microstructure (i.e., the properties of the basic unit cell and the interaction among basic cells) to determine mostly the properties of these metamaterials, much more than the physical and chemical characteristics of the constitutive fundamental materials used for their manufacturing (e.g., aluminum, steel, polyamide, etc.). The development of, for instance, devices in aerospace, naval and civil constructions, professional apparel, implants for surgical use and novel telescopes $[14,90]$ require the development of new high-performance materials.

A. Ciallella $(\varangle)$

Department of Civil, Construction-Architectural and Environmental Engineering, International Research Center M\&MoCS, University of L'Aquila, Via Giovanni Gronchi 18, 67100 L'Aquila, Italy

E-mail: alessandro.ciallella@univaq.it 
But how to develop new metamaterials? The idea can be to establish a priori the mechanical properties which one desires for its new material. To fix the properties which are to be demanded, one can state that a metamaterial is governed by some a priori chosen equations, and these equations determine its evolution. An effective choice to impose these equations may consist in choosing an action functional and by finding the evolution equations via the calculation of the corresponding Euler-Lagrange conditions. This deduction method has been used by D'Alembert, Lagrange, Piola [67] (see, for instance, the discussion in [29,30]) and more recently by Mindlin, Toupin $[69,94]$. The main advantages in using a variational approach consist, when using infinite-dimensional models, in the possibility of deducing immediately the class of boundary condition which are compatible with the bulk evolution equations (see [48-50,66]). It is possible to include dissipation effects starting either from a postulation based on the principle of virtual work, more general than the Least Action principle (see $[5,35,37,38,91]$ ), or from a postulation based on the Hamilton-Rayleigh principle, where a dissipation functional is added to the action functional (see, e.g., [33,43,46,85,92]). Once the mathematical model which one wants to use has been fixed, choosing Lagrange and possibly Rayleigh functions (as done in [51]), then the problem of the design of a metamaterial becomes a problem of synthesis. Subsequently, one has to find a microstructure for the searched mechanical system that, once homogenized (e.g., $[2,3,13,75,81])$, gives rise to an evolution governed exactly by these functions. The synthesis problem is not new in physics: Indeed, at least during the decades 1930-1950 this problem was topical for the industry of electric circuits. In fact, the analog computers were nothing else that the electrical synthesis of some ODEs or some PDEs (see [21,65]). The concepts developed by Kron were used more recently to synthesize piezoelectromechanical metamaterials, where the coupling with a given mechanical system is created via piezoelectric transducers $[56,68]$. In the context of purely mechanical metamaterials, the synthesis techniques have found many interesting applications (see, e.g., $[10,12,28,40,102])$. Given second gradient deformation energies, the problem is to find the microstructure of a metamaterial which is storing deformation energy correspondingly. Some solutions for the last specific problem can be found in $[4,41,86]$. More generally, one can try to get targeted materials basing, for instance, the demands: (i) on the mechanical properties of granular materials as done in [71]; (ii) by requiring some particular properties when damage occurs as in $[73,79,87]$; (iii) by designing some scaffolds for bone reconstruction as in [52]; (iv) by requiring that the microscopic structure exhibits some long-range interactions as in [42,64].

Important mathematical problems arise when trying to introduce "reduced-order" models, which are valid at a macroscale and are models which supply a simplified mathematical description of complex systems, by assuming a "direct" continuum description [58]. Also mathematically difficult is to study the case of metamaterials including fibers that can be assumed to be inextensible: The treatment, based on Pipkin seminal results $[76,77,89]$, has given interesting results, which show, however, how difficult is to study continua subjected to kinematical constraints [22,23,31,82]. Important numerical problems have to be faced to get reliable predictions about the mechanical behavior of the designed metamaterials. Most relevant effects seem to occur mainly in the nonlinear regimes, and correspondingly, the numerical algorithms to be used must be able to handle nonlinearity, bifurcation, loss of stability and similar phenomena [61,95,99].

Recently, synthesized materials have been designed to show exotic bulk-to-shear-moduli ratios (e.g., auxetic materials), negative compressibility transitions, unusual dynamic behavior for wave propagation. Also, materials with enhanced mechanical properties (robust buckling responses against structural perturbations), extreme strength-to-weight ratios, programmable shape transformations (for instance, exploiting elastic instabilities of the microstructure, like origami structures) and negative effective masses have been recently engineered. In many cases, these peculiar behaviors can be produced by taking advantage from the interaction among mechanical phenomena taking place at different length scales. A possible approach to deal with such multiscale mechanical interactions is represented by higher-order gradient continuum theories [15].

Pantographic fabrics could be a paradigmatic example to discuss some research perspectives that can be resulting in a progress of the knowledge of multiphysics and multiscale materials. Indeed, the intrinsic multiscale interactions present in this kind of materials lead to a second gradient continuum model description. Also, proper choices of the constituting materials (piezoelectric or flexoelectric, ionic polymer metal composite $[19,27])$ could easily lead to multiphysics behaviors as done in [45].

\section{Literature review}

Pantographic fabrics (see Fig. 1) are materials constituted by two orthogonal families of long beams, sometimes also referred to as fibers, interconnected at their intersection points by elastic pivots, i.e., small cylinders which allow for relative rotations at the expense of some deformation energy [36,39]. 

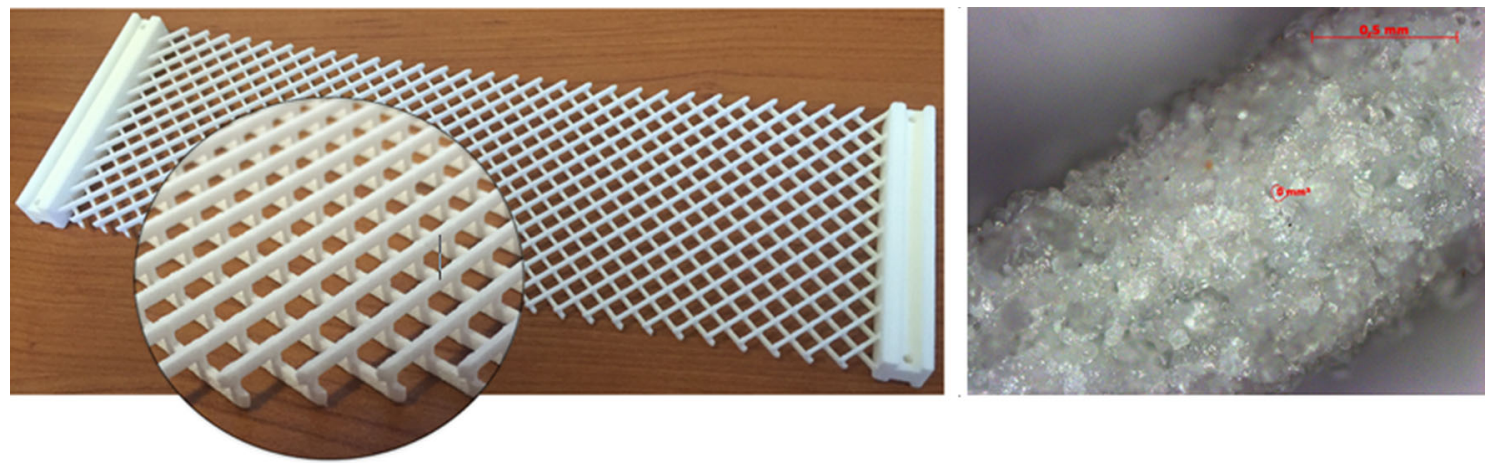

Fig. 1 A 3D printed pantographic fabric using PA2200 powder as constituting material (left). Magnification of a 3D printed polyamide short arm obtained by means of optical microscopy (right)
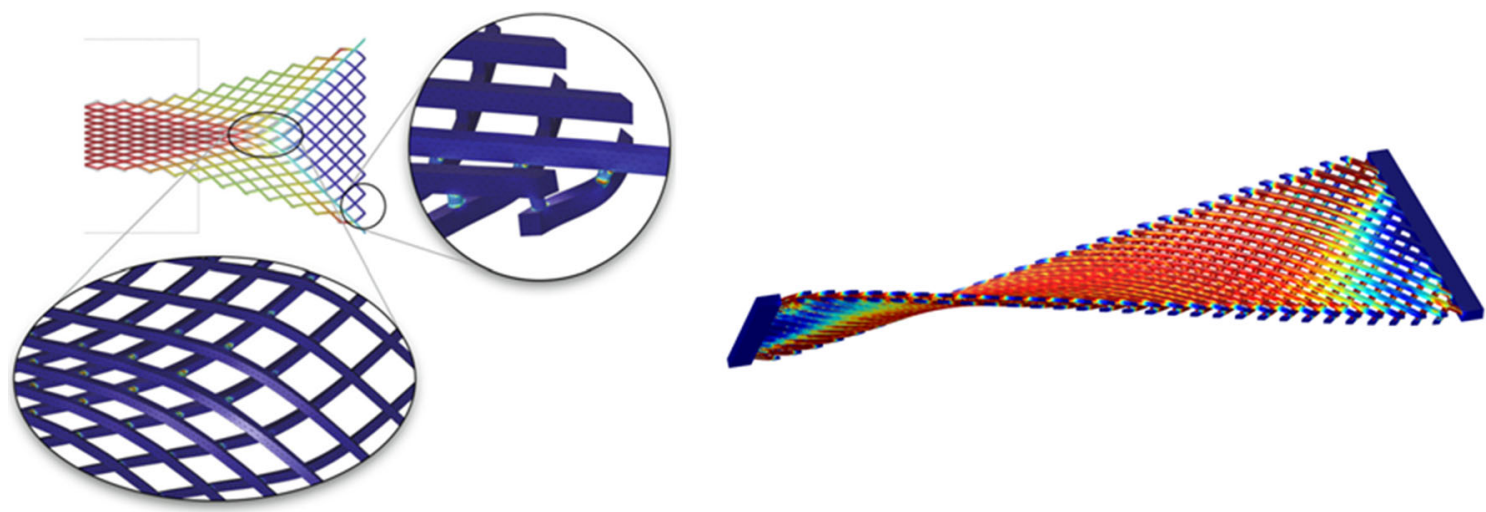

Fig. 2 2D top view with 3D view of zoomed details of the deformed shape of a pantographic fabric subject to a bias extension test (left) and 3D view of a pantographic specimen subject to twist (right). Numerical simulations were performed by means of the Cauchy continuum model. Cold colors are at the bottom of the color scale, while warm colors are at the top of the scale. Colors in the 2D view indicate the shear strain, i.e., deviation from being orthogonal of two fibers intersecting in a point, while colors in the $3 \mathrm{D}$ view indicate qualitatively the deformation energy

Pantographic fabrics have been proposed recently as a metamaterial which is well described at the macroscopic scale by second gradient continuum theories [34]. Fibers can be modeled as beams, possibly subjected to large deformations [11,54]. Pioneering papers [20,47,62,69,94] recognized the importance of understanding microscale mechanics in order to deduce macroscale behavior of continuum models. At the smallest scale, one can conceive of atomistic models to study the material behavior by investigating atomic interactions. The building block scales for atomistic models vary from 1 Åup to $50 \AA$. Analyses at this scale can in principle lead to high accuracy in results. However, granular materials like printed polyamide are still far to be dealt with atomistic modeling because of their complex composition which takes into account ionic, covalent and hydrogen bonds. Atomistic models are practically impossible at scales larger than few hundred atoms for complex material systems. The difficulty of atomic models is not only confined to their high computational cost, but more importantly, it is due to the impossibility of specifying the atomic structures of the myriad of material phases and interphases including their defects. Coarse-grained models such as molecular models, bead-spring models or discrete element models can incorporate microscale effects, resulting in a more advantageous method to deal with scales from the atomistic one $\sim 10^{-9} \mathrm{~m}$ to Cauchy continuum one $\sim 10^{-3} \mathrm{~m}$. In [53], full-scale 3D simulations at Cauchy continuum scale have been performed (see Fig. 2).

A coarser 2D discrete spring model resembling the pantographic structure (see Fig. 3) has been introduced in [96] (see also [18,100]). The blue/red rotational springs in Fig. 3 simulate the in-plane bending stiffness of fibers, the green rotational springs simulate the in-plane torsional stiffness of pivots, and the extensional springs simulate the extensional stiffness of fibers.

This last approach resulted to be very effective in describing in-plane deformations of the more refined 3D Cauchy model which, in turn, gives results almost overlapping with experimental ones [97,98]. This motivated a further coarsening: Starting from this discrete spring model, an asymptotic homogenization procedure (i.e., 

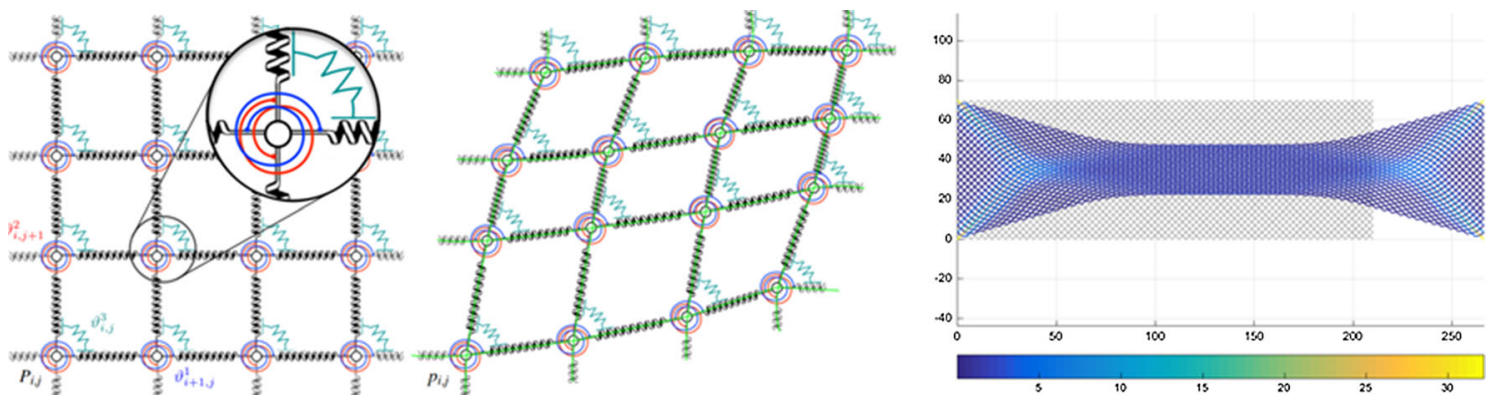

Fig. 3 Discrete spring two-dimensional model employed in [96] for the modeling of pantographic fabrics (left). Current shape of the spring model in a bias extension test (right) (color figure online)
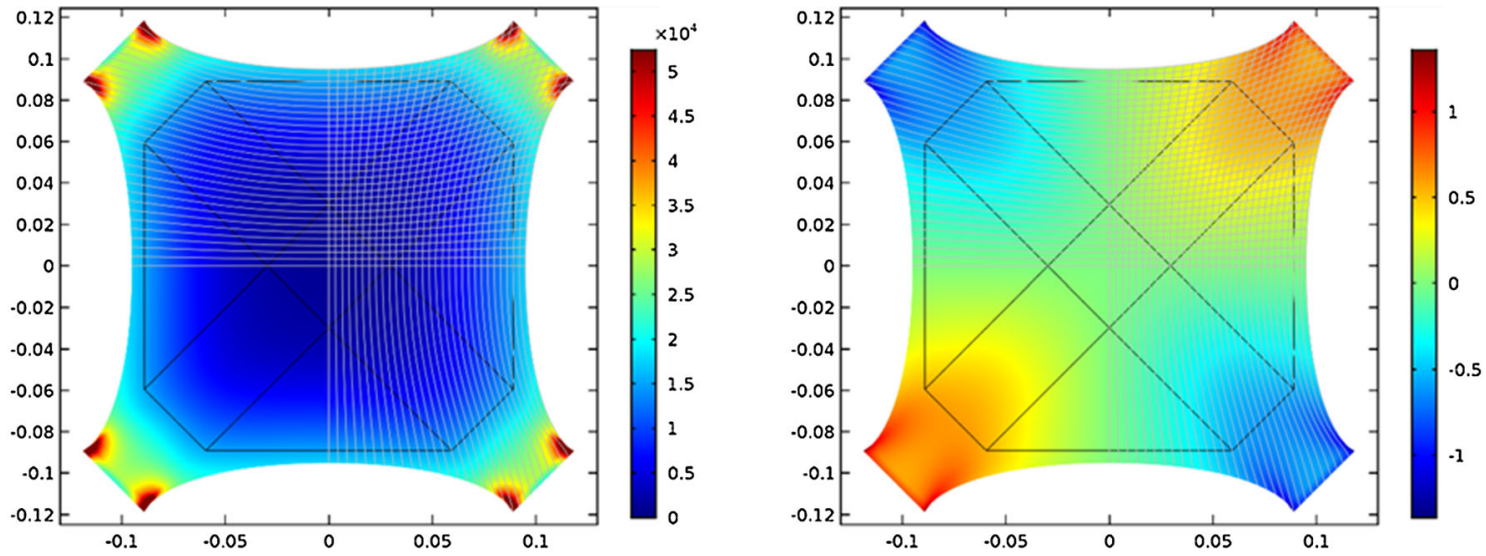

Fig. 4 Equilibrium shapes of pantographic fabrics subject to a bias bi-axial extension test. Simulations were performed using a 2D homogenized continuum model. Colors indicate the energy density on the left panel and the shear deformation on the right panel. Black solid lines indicate the reference configuration. Gray solid lines indicate material lines in the current configuration (color figure online)

assuming that the pitch of the fabrics tends to zero) has led to a second gradient continuum [32]. It is obtained that the static behavior of such a continuum is characterized by a deformation energy $U$ depending on the deformation gradient tensor $\mathrm{F}$ and its gradient $\nabla \mathrm{F}$ (this is the reason why, unlike Cauchy materials, pantographic fabrics are higher-gradient materials, and in particular, they are "second" gradient materials: $\nabla \mathrm{F}$ involves the second spatial derivative of the displacement $\chi$ ), both evaluated along the fiber directions $\mathbf{D}_{1}$ and $\mathbf{D}_{2}$ :

$$
\begin{aligned}
U(\chi(\cdot))= & \int_{\Omega} \sum_{\alpha} \frac{K_{\mathrm{e}}^{\alpha}}{2}\left(\left\|\mathrm{~F} \mathbf{D}_{\alpha}\right\|-1\right)^{2} \mathrm{~d} \Omega \\
& +\int_{\Omega} \sum_{\alpha} \frac{K_{\mathrm{b}}^{\alpha}}{2}\left[\frac{\nabla \mathrm{F}\left|\mathbf{D}_{\alpha} \otimes \mathbf{D}_{\alpha} \cdot \nabla \mathrm{F}\right| \mathbf{D}_{\alpha} \otimes \mathbf{D}_{\alpha}}{\left\|\mathrm{F} \mathbf{D}_{\alpha}\right\|^{2}}-\left(\frac{\mathrm{FD}_{\alpha}}{\left\|\mathrm{F} \mathbf{D}_{\alpha}\right\|} \cdot \frac{\nabla \mathrm{F} \mid \mathbf{D}_{\alpha} \otimes \mathbf{D}_{\alpha}}{\left\|\mathrm{F} \mathbf{D}_{\alpha}\right\|^{2}}\right)^{2}\right] \mathrm{d} \Omega \\
& +\int_{\Omega} \frac{K_{\mathrm{p}}^{\alpha}}{2}\left[\arcsin \left(\frac{\mathrm{FD}_{1}}{\left\|\mathrm{~F} \mathbf{D}_{1}\right\|} \cdot \frac{\mathrm{FD}_{2}}{\left\|\mathrm{~F} \mathbf{D}_{2}\right\|}\right)\right]^{2} \mathrm{~d} \Omega
\end{aligned}
$$

with $\alpha$ taking values 1 and 2 for the two families of fibers and $K_{\mathrm{e}}^{\alpha}, K_{b}^{\alpha}$ and $K_{\mathrm{p}}^{\alpha}$ being material stiffness related to fibers stretching, bending and pivots twisting (macroscopic shear), respectively. It is worth noting that the material parameters can be estimated numerically or experimentally as done in [26,57,63]. This model is computationally very efficient. Indeed, it can be solved numerically faster than a much more refined Cauchy model which is time-consuming because of the greater number of degrees of freedom (see Fig. 4).

Existence and uniqueness of weak solutions to the Euler-Lagrange equations for the above deformation energy have been proved by means of anisotropic Sobolev spaces in [44]. Results obtained with this continuum model are in very good agreement with experimental ones (see Fig. 5), meaning that during the homogenization process the unavoidable loss of information does not affect the relevant properties of the modeling. 


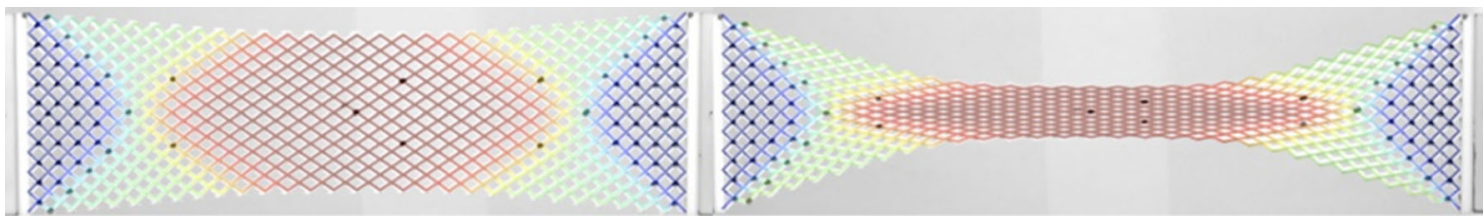

Fig. 5 Equilibrium shapes of pantographic fabrics subject to a bias extension test. Simulations performed using a 2D homogenized continuum model (deformed material lines) fit excellently the experimental data in the background even for large displacements

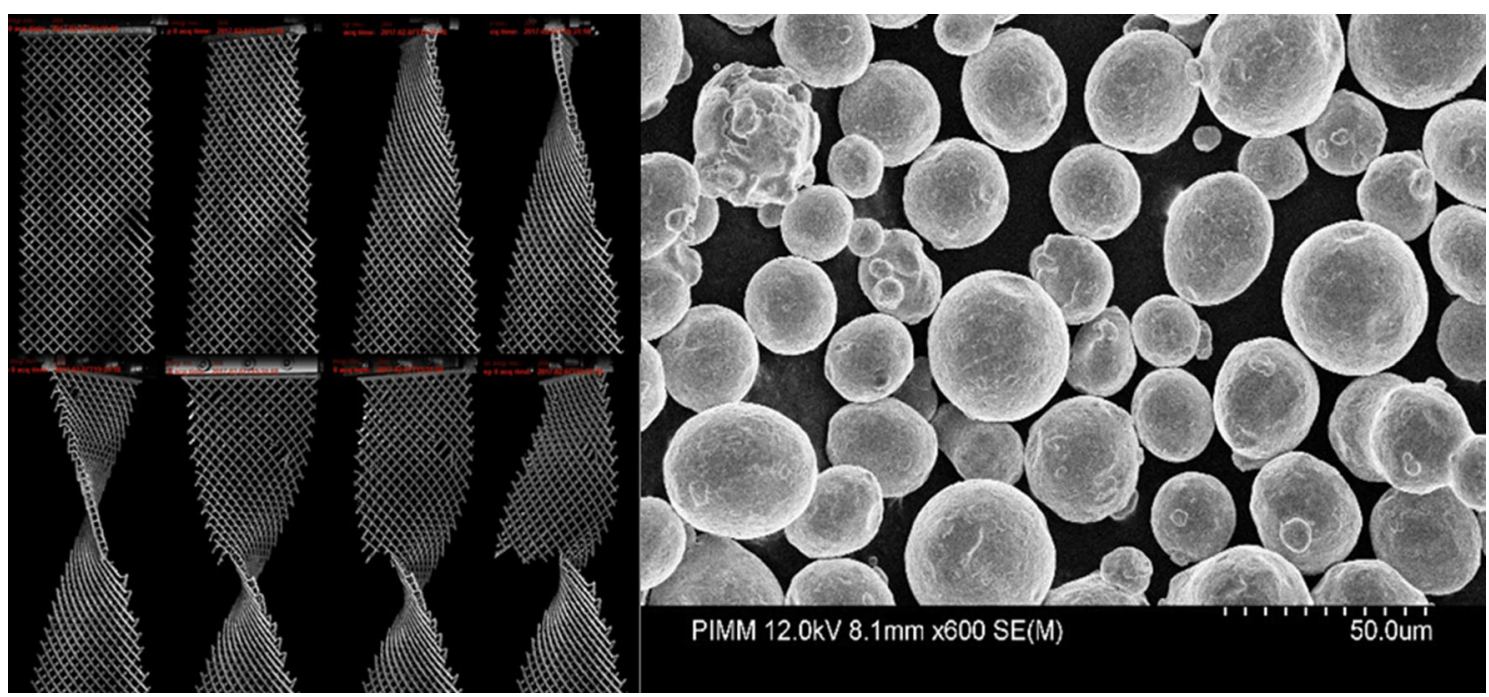

Fig. 6 Torsion test on aluminum pantographic fabrics (left). SEM magnification of aluminum powder used in its manufacturing (right)
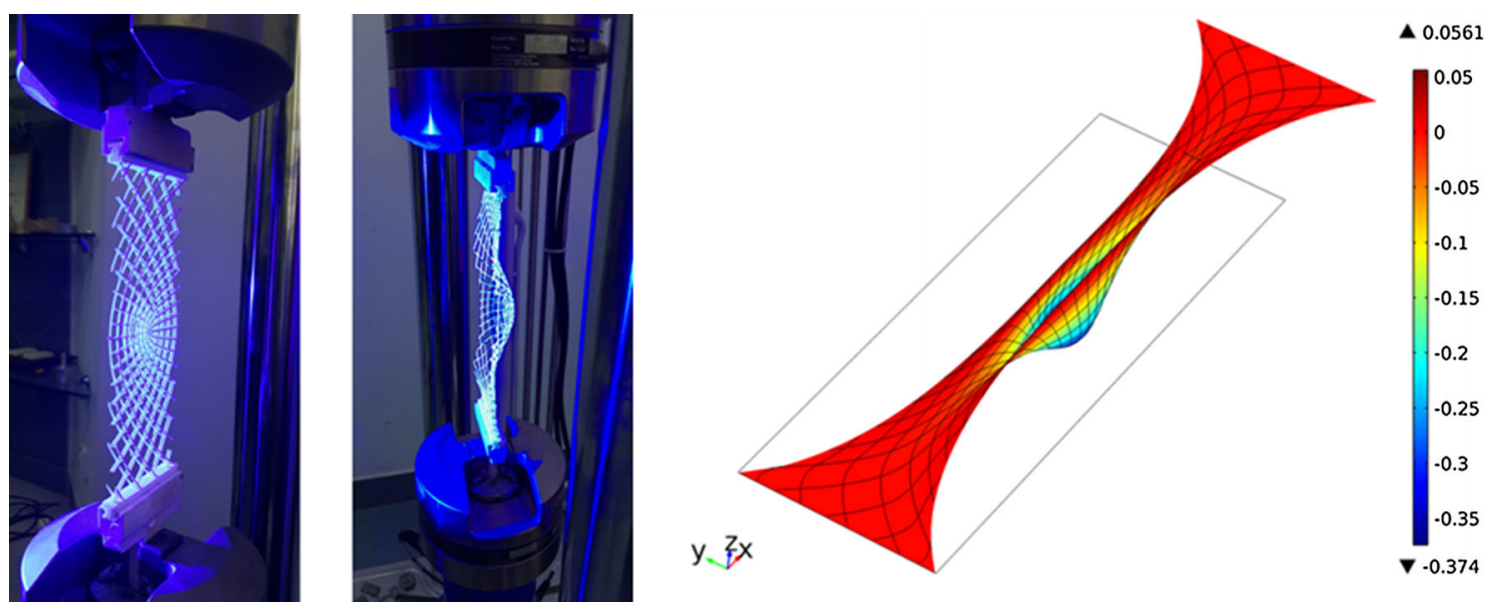

Fig. 7 Bias extension test of a pantographic fabric with non-straight (parabolic) fibers. Macroscopic (negative, as it is occurring in tension and not in compression) buckling (very exotic, as buckling is not usually observed in extension tests of standard plates) induced by microscopic buckling, i.e., by buckling of middle fibers subject to compression due to global Poisson effect

Further extensions to this continuum model in order to take into account out-of-plane motions with a reduced-order two-dimensional surface model moving in the space have been proposed in $[59,88]$. Such extended model has been employed in [9] in order to study buckling in pantographic fabrics subject to a shear test. Poynting effect reversal [70] has been recently observed in pantographic fabrics (see Fig. 6), as well as negative buckling effect (see Fig. 7), i.e., buckling due to critical extension (and not compression, as it is usual) load [55, 83,84]. 

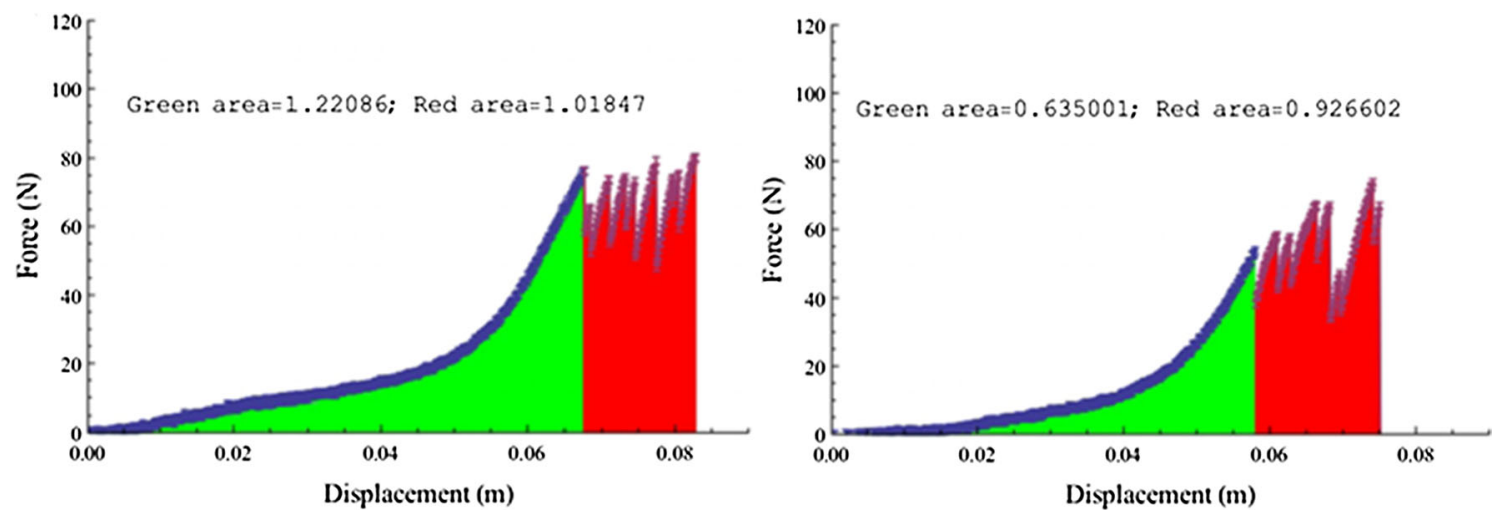

Fig. 8 Force-displacement diagrams for two bias extensions tests of pantographic fabrics (color figure online)

Pantographic structures proved to exhibit very interesting features. Regarding their static behavior, in addition to what has been shown so far, they exhibit a wide elastic regime (20-30\% elastic elongation, see the green regions in the force-displacement diagrams for two extension bias tests in Fig. 8), and while being compliant for small deformations, they stiffen extremely for large deformations. Therefore, they exhibit an advantageous strength-to-weight ratio $[34,80]$. Pantographic fabrics are very resilient. Indeed, after the first fiber/pivot rupture occurs (see the onset of the irreversible damaging regions colored in red), the loads that were acting on the ruptured structural member are redistributed over the adjacent members. After each fiber/pivot rupture (see the sudden force drops in Fig. 8), the structure is able to redistribute smartly the load so that it can carry forces comparable with (if not larger than) the elastic peak occurring before first fiber/pivot rupture. Moreover, the external energy needed to have the final failure of the pantographic fabrics after the onset of the irreversible damage phase (i.e., the area of the red regions in Fig. 8) is comparable with (if not larger than) the energy needed to cause the first fiber/pivot rupture (i.e., the green area of the green regions in Fig. 8).

\section{Future challenges in pantographic metamaterials research}

From prior works, it is well understood that continuum modeling is arguably the most suitable tool in order to deal with complex systems without resorting to computationally expensive descriptions that unavoidably require too many information about the considered system and that the theory of (incomplete) second gradient elasticity is needed in order to describe standard pantographic fabrics, as second gradient terms involved in the strain energy take into account the relevant contribution of in-plane bending of the constituting fibers to the peculiar phenomenology of pantographic fabrics [78].

A new class of pantographic fabrics has been proposed in [86]. Such a new kind of pantographic fabrics (i.e., pantographic fabrics whose constituting fibers are in turn pantographic slender systems, see Fig. 9) has been designed as a generalization of standard pantographic fabrics; indeed, its constituting fibers are not standard beams, i.e., they cannot be modeled as lines whose deformation energy depends only upon bending and elongation, but their deformation energy depends also upon the elongation gradient [7,72]. In the literature, this is the first example of material which can be modeled at a macroscopic scale by means of "complete" second gradient theories, meaning that all the pure second derivatives of the displacement field are appearing in the deformation energy. In other words, in this new kind of structures (in addition to second gradient contributions due to bending of constituting fibers) further second gradient contributions appear due to the differential elongation of constituting fibers. By looking at Fig. 9, it is clear that such a kind of new pantographic fabrics exhibits a wide class of zero-energy deformation modes, meaning that all the configurations in Fig. 9 are achieved without expending any external work. This is an extreme property, considering that for standard Cauchy materials only rigid motions are entailing zero energy.

Future challenges in pantographic metamaterials research include at least the following three objectives: as the first, to formulate coarse-scale nonlinear second gradient continuum models (this would be a novel accomplishments, as the previous literature on such a kind of new structure deals only with linear models, i.e., models in which the strain energy density depends linearly upon the deformation gradient and its gradient), describing such a new class of pantographic metamaterials on the ground of finer-scale descriptions through homogenization techniques, using the fibers' equations obtained in [7] and proceeding in a way analogous 
to [32]; second, to implement finite element analyses with shape functions possessing higher regularity (e.g., NURBS elements) or employing mixed formulations to perform simulations with the above-formulated models $[16,17,24,60,74,101]$; and third, to validate and verify the derived models through the acquisition and analysis of experimental data.

\section{Methodological issues}

Following the approach presented in [32] and relying on previous results [6-8] for what concerns a single pantographic fiber, a homogenization procedure shall be carried out in order to find the nonlinear counterpart of the energy found in the seminal work [86]. By means of asymptotic expansions of the kinematic parameters with respect to the size of the basic unit cell, it is possible to determine (at the leading order in the size of the basic unit cell and, then, in the limit of this size tending to zero) the energy of the considered system. Assuming that, for a fixed length of the macroscopic specimen, the size of the basic unit cell tends to zero is equivalent to stating that the material is made up of a multitude of cells and, therefore, that the continuum hypothesis is legitimate.

\subsection{Model predictions and design of experiments}

Model predictions shall be computed by means of numerical simulations. The finite element computation of higher-gradient continua requires care for some specific issues. The most relevant issue is that higher-gradient continua require higher regularity of the shape functions employed in finite element methods [1]. Ad hoc solutions shall be adopted within working environments provided either by open-source platforms (FEniCS project) or proprietary platforms (Abaqus, Comsol Multiphysics, etc.). Experiments shall be conceived after numerical evidence has shown that they are suitable to determine characteristic properties of the considered
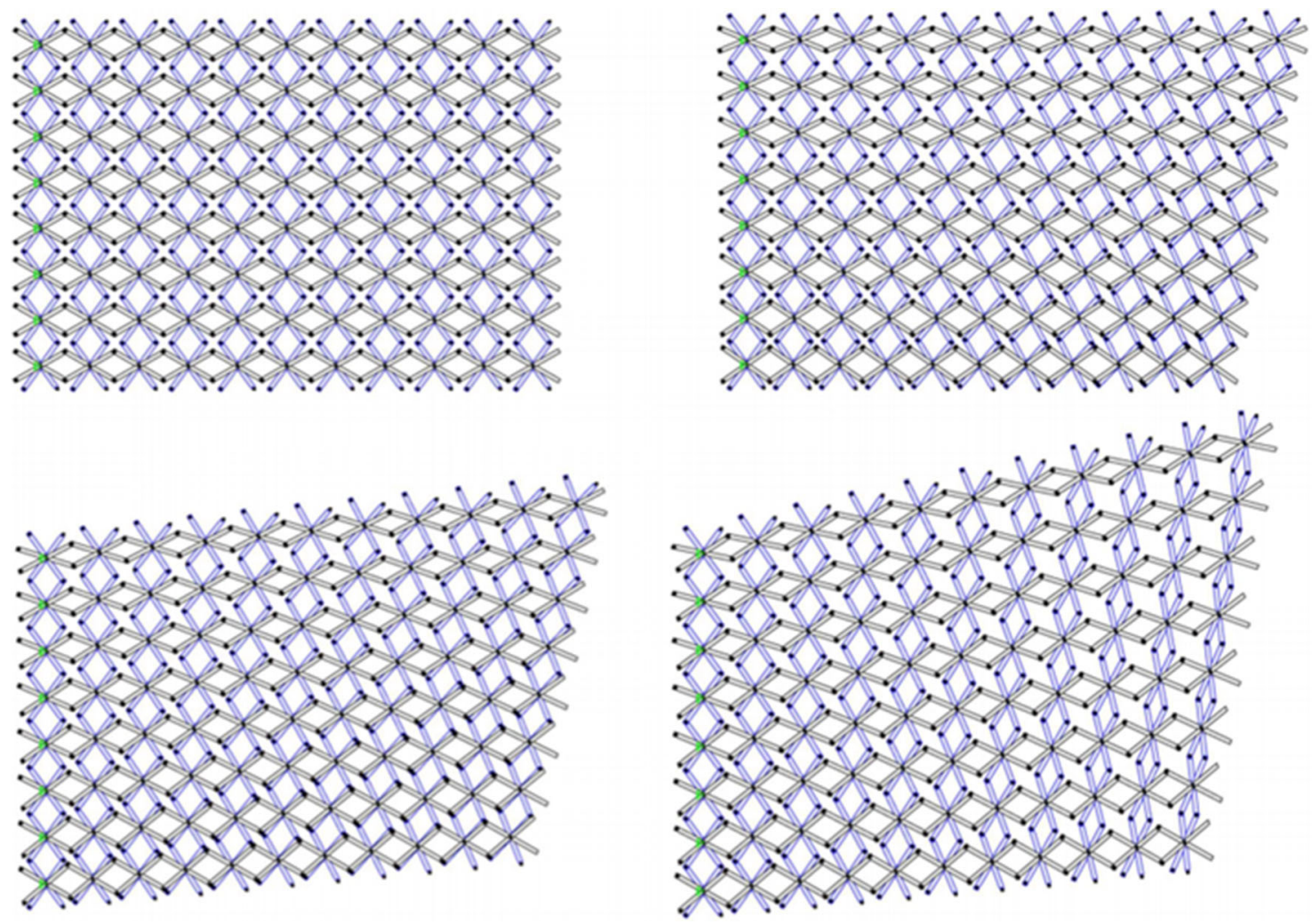

Fig. 9 Floppy modes of pantographic fabrics with pantographic beams as constituting fibers 
materials. Figure 10 shows experiments performed upon standard pantographic fabrics; the same loading apparatus can be used for the new pantographic metamaterial.

\subsection{Data collection and analysis for model validation}

Since very large displacement levels occur, digital image correlation (DIC, see [25]) is a natural choice for the measurement technique (see Figs. 11, 12). A set of such deformation measurements (training set), together with force-displacement diagrams obtained by means of the load machine transducer, shall be used in order to fit the parameters of the differently refined models. Another set of measurements shall be used as test set for model validation. DIC analyses for establishing deformations in pantographic fabrics have already been employed. In Fig. 11, different meshes for DIC applied to pantographic fabrics are shown. Standard meshes made of 3-noded triangles with linear shape functions (i.e., T3-DIC) have shown to be not compatible with the pantograph structure, and therefore, other kind of meshing have been explored [93].

\subsection{Manufacturing of specimens}

The preprinting phase is mainly based on the use of a computer. First of all, one has to design the model by means of a CAD software (see Fig. 13, where a tentative design of the new pantographic structure has been attempted). Then, one has to prepare the process of selective laser sintering (SLS) 3D printing with all the setting parameters (preheating temperature, laser power, bed cooling time, etc.), and finally, the arrangement in the virtual printing chamber is required. After printing, especially in SLS technology, it is necessary to wait until all the objects are cooled down in order to reduce material contractions. In the end, the cleaning process is carried out. It is done, for example, using air pressure, abrasive blasting or ultrasonic washers. Another aspect to
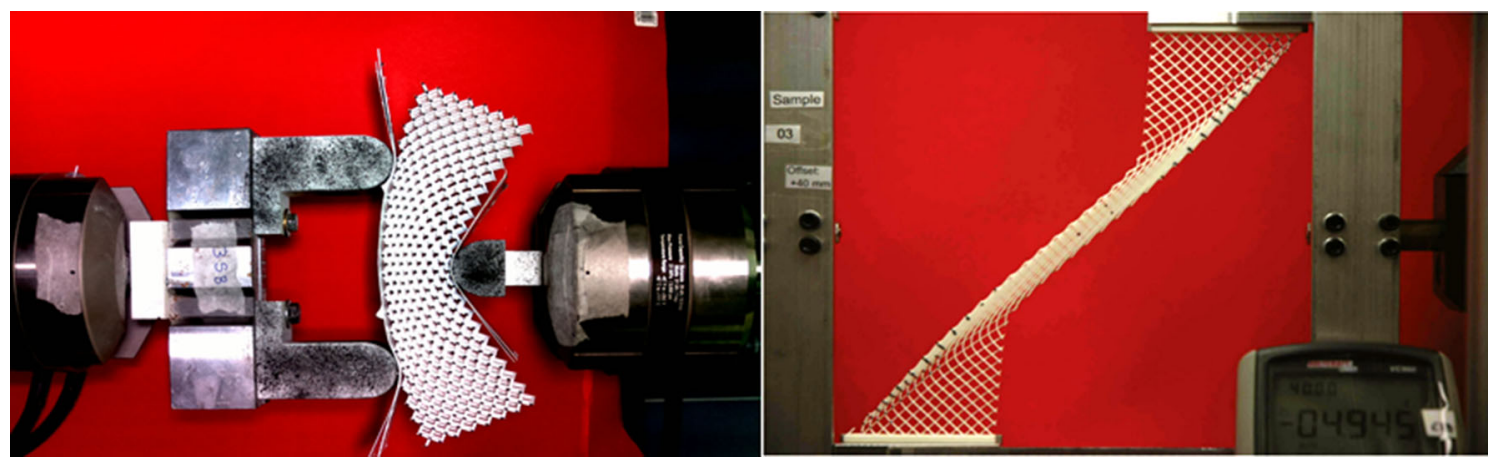

Fig. 10 Three-point test (left) and shear test (right) on pantographic metamaterials printed with PA2200

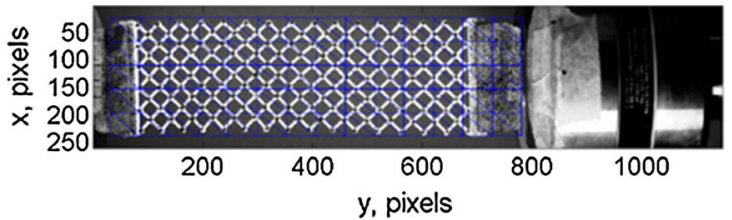

(a) mesh 1

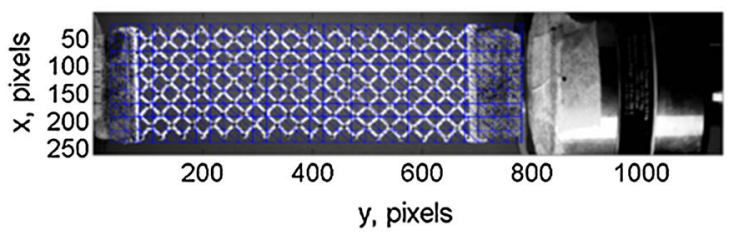

(c) mesh 3

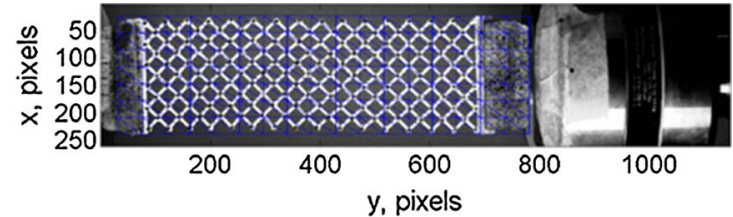

(b) mesh 2

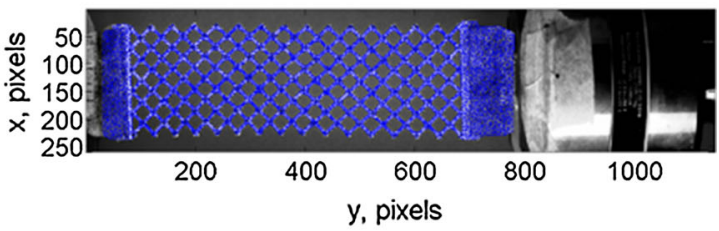

(d) mesh 4

Fig. 11 Finite element meshes overlaid with the gray level picture of the reference configuration 


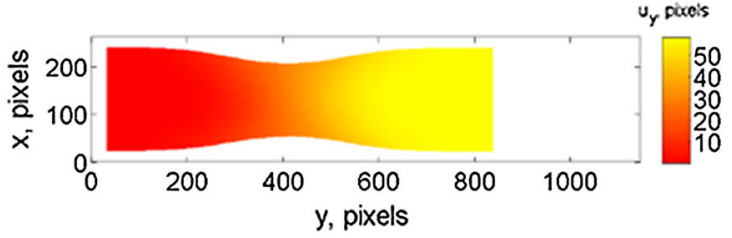

(a)

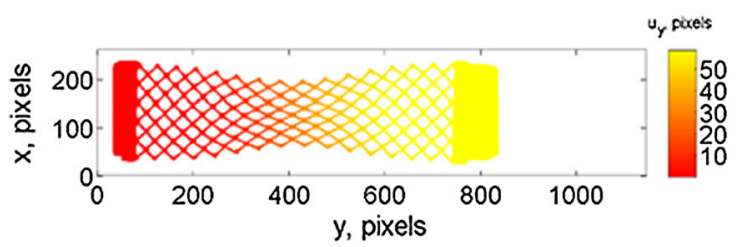

(c)

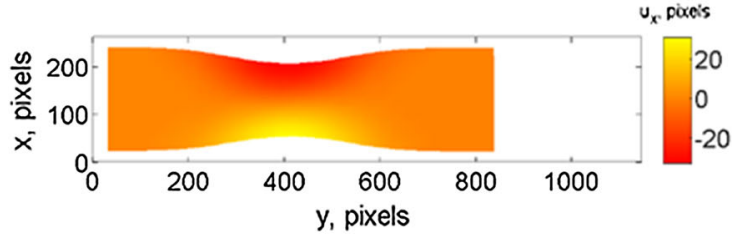

(b)

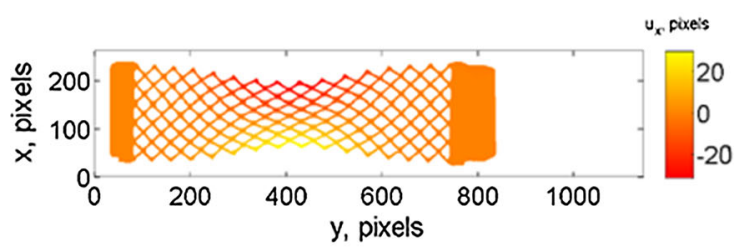

(d)

Fig. 12 Longitudinal (a-c) and transverse (b-d) displacement fields measured with meshes $3(\mathbf{a}, \mathbf{b})$ and $4(\mathbf{c}, \mathbf{d})$. The fields are shown on the deformed configuration

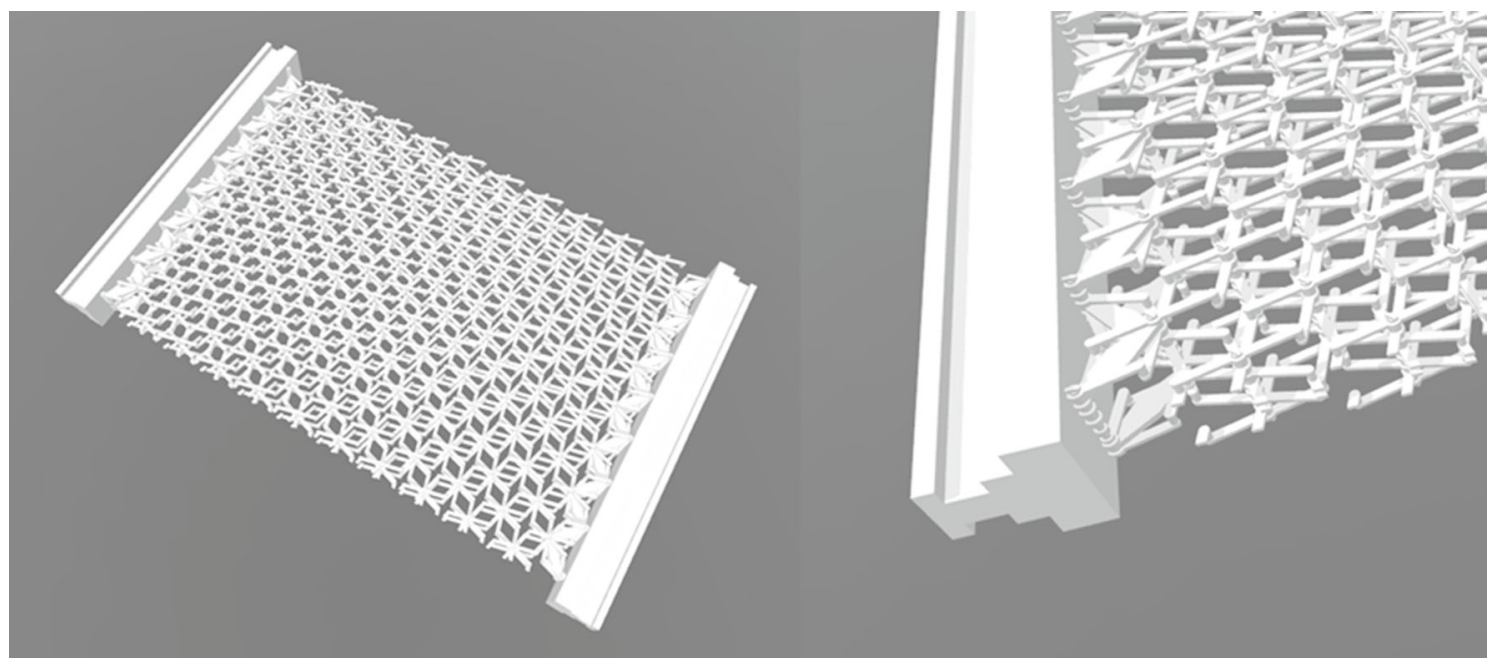

Fig. 13 3D view of a tentative CAD model of the new pantographic structure

account for in SLS 3D printing is the orientation of given parts in the printing chamber. A wrong orientation of the model, especially when the model includes moving parts (like in the case of the new pantographic fabrics), can affect functionality or make a specimen completely useless. In conclusion, a study should be carried out in order to find the optimal setting for specimens manufacturing and the optimal geometric properties of the CAD model.

\section{Conclusion}

To summarize, some of the future challenging steps in pantographic metamaterials research are listed below.

Optimal design and print settings (using SLS printers), and best post-press and prepress practices (e.g., cleaning and cooling procedures to reduce sample damage and/or prestress, preparation and powder composition) need to be defined to provide technologically and scientifically useful information to those aiming to improve SLS 3D printing quality. It will require the production of CAD files working with the most widely used software and printers, as well as the actual production of prototypes expected to have exotic and interesting properties (most likely useful for some applications mentioned at the beginning). Further enhancement would be brought about by the formulation of higher-gradient or microstructured continuum models by means of homogenization techniques starting from the synthesized microstructures. Indeed, the continuum models are preferable from a computational point of view, so they allow a better mechanical understanding of 
involved phenomena and a rapid design process. The numerical calculations necessary for design and predicting will require the implementation of finite element analyses with shape functions possessing higher regularity (e.g., NURBS elements) or employing mixed formulations or isogeometric schemes to deal with the above-formulated models. Finally, it will be required to work in the development of experimental protocols and tests useful to characterize the constitutive parameters of the new pantographic fabrics, and to test and advance state-of-the art DIC techniques (e.g., mechanics-based DIC).

As a concluding remark, one should not believe that pantographic structures are a too specific example of metamaterial. In fact, we conjecture that the more general problem of synthesis of higher-gradient microstructured continua [30] could be tackled by building elementary periodic cells constituted by a finite set of substructures, interconnected in a suitable graph. This should provide a parallel result to a corresponding one in circuit theory: Each passive linear circuit can be synthesized using capacitors, inductors, transformers and resistors as elementary units.

Acknowledgements The author thanks Dr. Emilio Barchiesi for introducing him to the topic and for the stimulating discussions that led to the present work.

\section{References}

1. Abali, B.E., Müller, W.H., dell'Isola, F.: Theory and computation of higher gradient elasticity theories based on action principles. Arch. Appl. Mech. 87, 1495-1510 (2017)

2. Abdoul-Anziz, H., Seppecher, P.: Homogenization of periodic graph-based elastic structures. J. l'École Polytech. Math. 5, 259-288 (2018)

3. Abdoul-Anziz, H., Seppecher, P.: Strain gradient and generalized continua obtained by homogenizing frame lattices. Math. Mech. Complex Syst. 6(3), 213-250 (2018)

4. Alibert, J.-J., Seppecher, P., dell'Isola, F.: Truss modular beams with deformation energy depending on higher displacement gradients. Math. Mech. Solids 8(1), 51-73 (2003)

5. Altenbach, H., Ochsner, A. (eds.): State of the Art and Future Trends in Material Modeling. Springer, Berlin (2019)

6. Barchiesi, E., dell'Isola, F., Hild, F., et al.: Two-dimensional continua capable of large elastic extension in two independent directions: asymptotic homogenization, numerical simulations and experimental evidence. Mech. Res. Commun. 103, $103466(2020)$

7. Barchiesi, E., dell'Isola, F., Laudato, M., et al.: A 1D continuum model for beams with pantographic microstructure: asymptotic micro-macro identification and numerical results. In: dell'Isola, F., Eremeyev, A., Porubov, A. (eds.) Advances in Mechanics of Microstructured Media and Structures. Advanced Structured Materials, vol. 87. Springer, Cham (2018)

8. Barchiesi, E., Eugster, S.R., dell'Isola, F., et al.: Large in-plane elastic deformations of bi-pantographic fabrics: asymptotic homogenization and experimental validation. Math. Mech. Solids 25(3), 739-767 (2020)

9. Barchiesi, E., Ganzosch, G., Liebold, C., et al.: Out-of-plane buckling of pantographic fabrics in displacement-controlled shear tests: experimental results and model validation. Contin. Mech. Thermodyn. 31, 33-45 (2019)

10. Barchiesi, E., Spagnuolo, M., Placidi, L.: Mechanical metamaterials: state of the art. Math. Mech. Solids 24(1), 212-234 (2018)

11. Baroudi, D., Giorgio, I., Battista, A., et al.: Nonlinear dynamics of uniformly loaded elastica: experimental and numerical evidence of motion around curled stable equilibrium configurations. ZAMM J. Appl. Math. Mech. 99(7), e201800121 (2019)

12. Bersani, A.M., Della Corte, A., Piccardo, G., et al.: An explicit solution for the dynamics of a taut string of finite length carrying a traveling mass: the subsonic case. Z. Angew. Math. Phys. 67(4), 108 (2016)

13. Boutin, C., Giorgio, I., Placidi, L.: Linear pantographic sheets: asymptotic micro-macro models identification. Math. Mech. Complex Syst. 5(2), 127-162 (2017)

14. Buffa, F., Causin, A., Cazzani, A., et al.: The Sardinia Radio Telescope: a comparison between close-range photogrammetry and finite element models. Math. Mech. Solids 22(5), 1005-1026 (2017)

15. Carcaterra, A., dell'Isola, F., Esposito, R., et al.: Macroscopic description of microscopically strongly inhomogenous systems: a mathematical basis for the synthesis of higher gradients metamaterials. Arch. Ration. Mech. Anal. 218, 1239$1262(2015)$

16. Cazzani, A., Malagú, M., Turco, E.: Isogeometric analysis of plane-curved beams. Math. Mech. Solids 21(5), 562-577 (2016)

17. Cazzani, A., Malagú, M., Turco, E., et al.: Constitutive models for strongly curved beams in the frame of isogeometric analysis. Math. Mech. Solids 21(2), 182-209 (2016)

18. Challamel, N., Kocsis, A., Wang, C.M.: Discrete and non-local elastica. Int. J. Non-Linear Mech. 77, $128-140$ (2015)

19. Chróścielewski, J., Schmidt, R., Eremeyev, V.A.: Nonlinear finite element modeling of vibration control of plane rod-type structural members with integrated piezoelectric patches. Contin. Mech. Thermodyn. 31, 147-188 (2019)

20. Cosserat, E., Cosserat, F.: Théorie des corps déformables (1909)

21. Crandall, S.H., Karnopp, D.C., Kurtz, E.F., et al.: Dynamics of Mechanical and Electromechanical Systems. McGraw-Hill, New York (1968)

22. Cuomo, M., dell'Isola, F., Greco, L.: Simplified analysis of a generalized bias test for fabrics with two families of inextensible fibres. Z. Angew. Math. Phys. 67, 61 (2016) 
23. Cuomo, M., dell'Isola, F., Greco, L., et al.: First versus second gradient energies for planar sheets with two families of inextensible fibres: investigation on deformation boundary layers, discontinuities and geometrical instabilities. Compos. B Eng. 115, 423-448 (2017)

24. Cuomo, M., Greco, L.: An implicit strong $G^{1}$-conforming formulation for the analysis of the Kirchhoff plate model. Contin. Mech. Thermodyn. (2018). https://doi.org/10.1007/s00161-018-0701-3

25. Curt, J., Capaldo, M., Hild, F., et al.: Optimal digital color image correlation. Opt. Lasers Eng. 127, 105896 (2020)

26. De Angelo, M., Barchiesi, E., Giorgio, I., et al.: Numerical identification of constitutive parameters in reduced-order bidimensional models for pantographic structures: application to out-of-plane buckling. Arch. Appl. Mech. 89(7), 1333-1358 (2019)

27. Del Bufalo, G., Placidi, L., Porfiri, M.: A mixture theory framework for modeling the mechanical actuation of ionic polymer metal composites. Smart Mater. Struct. 17, 4 (2008)

28. El Sherbiny, M.G., Placidi, L.: Discrete and continuous aspects of some metamaterial elastic structures with band gaps. Arch. Appl. Mech. 88(10), 1725-1742 (2018)

29. dell'Isola, F., Andreaus, U., Placidi, L.: At the origins and in the vanguard of peridynamics, non-local and higher-gradient continuum mechanics: an underestimated and still topical contribution of Gabrio Piola. Math. Mech. Solids 20(8), 887-928 (2015)

30. dell'Isola, F., Della Corte, A., Giorgio, I.: Higher-gradient continua: the legacy of Piola, Mindlin, Sedov and Toupin and some future research perspectives. Math. Mech. Solids 22(4), 852-872 (2017)

31. dell'Isola, F., Della Corte, A., Greco, L., et al.: Plane bias extension test for a continuum with two inextensible families of fibers: a variational treatment with Lagrange multipliers and a perturbation solution. Int. J. Solids Struct. 81, 1-12 (2016)

32. dell'Isola, F., Giorgio, I., Pawlikowski, M., et al.: Large deformations of planar extensible beams and pantographic lattices: heuristic homogenization, experimental and numerical examples of equilibrium. Proc. R. Soc. A 472, 20150790 (2016)

33. dell'Isola, F., Madeo, A., Seppecher, P.: Boundary conditions at fluid-permeable interfaces in porous media: a variational approach. Int. J. Solids Struct. 46(17), 3150-3164 (2009)

34. dell'Isola, F., Lekszycki, T., Pawlikowski, M., et al.: Designing a light fabric metamaterial being highly macroscopically tough under directional extension: first experimental evidence. Z. Angew. Math. Phys. 66, 3473-3498 (2015)

35. dell'Isola, F., Seppecher, P.: Edge contact forces and quasi-balanced power. Meccanica 32, 33-52 (1997)

36. dell'Isola, F., Seppecher, P., Alibert, J.J., et al.: Pantographic metamaterials: an example of mathematically driven design and of its technological challenges. Contin. Mech. Thermodyn. 31, 851-884 (2019)

37. dell'Isola, F., Seppecher, P., Madeo, A.: How contact interactions may depend on the shape of Cauchy cuts in Nth gradient continua: approach "á la D’Alembert". Z. Angew. Math. Phys. 63, 1119-1141 (2012)

38. dell'Isola, F., Seppecher, P., Placidi, L. et al.: Least action and virtual work principles for the formulation of generalized continuum models, Chap. 8. In: Dell'Isola, F., Steigmann D. (eds.) Discrete and Continuum Models for Complex Metamaterials (pp. 327-394). Cambridge University Press, Cambridge (2020). https://doi.org/10.1017/9781316104262.010

39. dell'Isola, F., Seppecher, P., Spagnuolo, M., et al.: Advances in pantographic structures: design, manufacturing, models, experiments and image analyses. Contin. Mech. Thermodyn. 31, 1231-1282 (2019)

40. dell'Isola, F., Steigmann, D.J. (eds.): Discrete and Continuum Models for Complex Metamaterials. Cambridge University Press, Cambridge (2020)

41. dell'Isola, F., Steigmann, D., Della Corte, A.: Synthesis of fibrous complex structures: designing microstructure to deliver targeted macroscale response. Appl. Mech. Rev. 67(6), 060804 (2015). https://doi.org/10.1115/1.4032206

42. Diyaroglu, C., Oterkus, E., Oterkus, S., et al.: Peridynamics for bending of beams and plates with transverse shear deformation. Int. J. Solids Struct. 69, 152-168 (2015)

43. Eremeyev, V.A., Altenbach, H.: Rayleigh variational principle and vibrations of prestressed shells. Shell Struct. Theory Appl. 3, 285-288 (2014)

44. Eremeyev, V.A., dell'Isola, F., Boutin, C., et al.: Linear pantographic sheets: existence and uniqueness of weak solutions. J. Elast. 132, 175-196 (2018)

45. Eremeyev, V.A., Ganghoffer, J.-F., Konopińska-Zmysłowska, V., et al.: Flexoelectricity and apparent piezoelectricity of a pantographic micro-bar. Int. J. Eng. Sci. 149, 103213 (2020). https://doi.org/10.1016/j.ijengsci.2020.103213

46. Eremeyev, V.A., Lebedev, L.P., Cloud, M.J.: The Rayleigh and Courant variational principles in the six-parameter shell theory. Math. Mech. Solids 20(7), 806-822 (2015)

47. Eringen A.C.: Theory of micropolar elasticity. In: Microcontinuum Field Theories. Springer, New York (1999). https://doi. org/10.1007/978-1-4612-0555-5_5

48. Eugster, S.R., Dell'Isola, F.: Exegesis of the introduction and sect. I from "fundamentals of the mechanics of continua". ZAMM J. Appl. Math. Mech. 97(4), 477-506 (2017)

49. Eugster, S.R., Dell'Isola, F.: Exegesis of sect. II and III. A from "fundamentals of the mechanics of continua". ZAMM J. Appl. Math. Mech. 98(1), 31-68 (2018)

50. Eugster, S.R., Dell'Isola, F.: Exegesis of sect. III. B from "fundamentals of the mechanics of continua". ZAMM J. Appl. Math. Mech. 98(1), 69-105 (2018)

51. Eugster, S., dell'Isola, F., Steigmann, D.: Continuum theory for mechanical metamaterials with a cubic lattice substructure. Math. Mech. Complex Syst. 7(1), 75-98 (2019)

52. George, D., Allena, R., Remond, Y.: A multiphysics stimulus for continuum mechanics bone remodeling. Math. Mech. Complex Syst. 6(4), 307-319 (2018)

53. Giorgio, I.: Numerical identification procedure between a micro-Cauchy model and a macro-second gradient model for planar pantographic structures. Z. Angew. Math. Phys. 67, 95 (2016)

54. Giorgio, I.: A discrete formulation of Kirchhoff rods in large-motion dynamics. Mech. Solids, Math (2020). https://doi.org/ 10.1177/1081286519900902

55. Giorgio, I., Della Corte, A., dell'Isola, F., et al.: Buckling modes in pantographic lattices. C. R. Mec. 344, 487-501 (2016)

56. Giorgio, I., Galantucci, L., Della Corte, A., et al.: Piezo-electromechanical smart materials with distributed arrays of piezoelectric transducers: current and upcoming applications. Int. J. Appl. Electromagn. Mech. 47(4), 1051-1084 (2015) 
57. Giorgio, I., Harrison, P., dell'Isola, F., et al.: Wrinkling in engineering fabrics: a comparison between two different comprehensive modelling approaches. Proc. R. Soc. A 474, 20180063 (2018)

58. Giorgio, I., Rizzi, N.L., Andreaus, U., et al.: A two-dimensional continuum model of pantographic sheets moving in a 3D space and accounting for the offset and relative rotations of the fibers. Math. Mech. Complex Syst. 7(4), 311-325 (2019)

59. Giorgio, I., Rizzi, N.L., Turco, E.: Continuum modelling of pantographic sheets for out-of-plane bifurcation and vibrational analysis. Proc. R. Soc. A 473, 20170636 (2017)

60. Greco, L., Cuomo, M., Contrafatto, L.: Two new triangular G1-conforming finite elements with cubic edge rotation for the analysis of Kirchhoff plates. Comput. Methods Appl. Mech. Eng. 356, 354-386 (2019)

61. Greco, L., Giorgio, I., Battista, A.: In plane shear and bending for first gradient inextensible pantographic sheets: numerical study of deformed shapes and global constraint reactions. Math. Mech. Solids 22(10), 1950-1975 (2017)

62. Green, A.E., Rivlin, R.S.: Multipolar continuum mechanics. Arch. Ration. Mech. Anal. 17, 113-147 (1964)

63. Harrison, P.: Modelling the forming mechanics of engineering fabrics using a mutually constrained pantographic beam and membrane mesh. Compos. A Appl. Sci. Manuf. 81, 145-157 (2016)

64. Javili, A., Morasata, R., Oterkus, E., et al.: Peridynamics review. Math. Mech. Solids 24(11), 3714-3739 (2019)

65. Kron, G.: Equivalent circuits of the elastic field. J. App, Mech. 11, 149-161 (1944)

66. Kroner, E.: Mechanics of Generalized Continua. Springer, Berlin (1968)

67. Lagrange, J.L.: Mécanique analytique, vol. 1. Mallet-Bachelier, Paris (1853)

68. Maurini, C., dell'Isola, F., Del Vescovo, D.: Comparison of piezoelectronic networks acting as distributed vibration absorbers. Mech. Syst. Signal Process. 18(5), 1243-1271 (2004)

69. Mindlin, R.D.: Micro-structure in linear elasticity. Arch. Ration. Mech. Anal. 16, 51-78 (1964)

70. Misra, A., Lekszycki, T., Giorgio, I., et al.: Pantographic metamaterials show atypical Poynting effect reversal. Mech. Res. Commun. 89, 6-10 (2018)

71. Misra, A., Poorsolhjouy, P.: Identification of higher-order elastic constants for grain assemblies based upon granular micromechanics. Math. Mech. Complex Syst. 3(3), 285-308 (2015)

72. Nejadsadeghi, N., De Angelo, M., Drobnicki, R., et al.: Parametric experimentation on pantographic unit cells reveals local extremum configuration. Exp. Mech. 59, 927-939 (2019)

73. Nguyen, T.H., Niiranen, J.: A second strain gradient damage model with a numerical implementation for quasi-brittle materials with micro-architectures. Math. Mech. Solids 25(3), 515-546 (2020)

74. Niiranen, J., Balobanov, V., Kiendl, J., et al.: Variational formulations, model comparisons and numerical methods for Euler-Bernoulli micro-and nano-beam models. Math. Mech. Solids 24(1), 312-335 (2019)

75. Pideri, C., Seppecher, P.: A second gradient material resulting from the homogenization of an heterogeneous linear elastic medium. Contin. Mech. Thermodyn. 9, 241-257 (1997)

76. Pipkin, A.C.: Equilibrium of Tchebychev nets. In: The Breadth and Depth of Continuum Mechanics, pp. 287-303. Springer, Berlin (1986). https://doi.org/10.1007/978-3-642-61634-1_12

77. Pipkin, A.C.: Catenary deformations of inextensible networks. J. Eng. Math. 28(5), 401-406 (1994)

78. Placidi, L., Andreaus, U., Giorgio, I.: Identification of two-dimensional pantographic structure via a linear D4 orthotropic second gradient elastic model. J. Eng. Math. 103, 1-21 (2017)

79. Placidi, L., Barchiesi, E., Misra, A.: A strain gradient variational approach to damage: a comparison with damage gradient models and numerical results. Math. Mech. Complex Syst. 6(2), 77-100 (2018)

80. Placidi, L., Barchiesi, E., Turco, E., et al.: A review on 2D models for the description of pantographic fabrics. Z. Angew. Math. Phys. 67, 121 (2016)

81. Placidi, L., dell'Isola, F., Barchiesi, E.: Heuristic homogenization of Euler and pantographic beams. In: Picu, C., Ganghoffer, J.F. (eds.) Mechanics of Fibrous Materials and Applications. CISM International Centre for Mechanical Sciences (Courses and Lectures), vol. 596. Springer, Cham (2020)

82. Placidi, L., Greco, L., Bucci, S., et al.: A second gradient formulation for a 2D fabric sheet with inextensible fibres. Z. Angew. Math. Phys. 67, 114 (2016)

83. Scerrato, D., Giorgio, I., Rizzi, N.L.: Three-dimensional instabilities of pantographic sheets with parabolic lattices: numerical investigations. Z. Angew. Math. Phys. 67, 53 (2016)

84. Scerrato, D., Giorgio, I.: Equilibrium of two-dimensional cycloidal pantographic metamaterials in three-dimensional deformations. Symmetry 11, 1523 (2019)

85. Seliger, R.L., Whitham, G.B.: Variational principles in continuum mechanics. Proc. R. Soc. Lond. A 305(1480), 1-25 (1968)

86. Seppecher, P., Alibert, J.-J., dell'Isola, F.: Linear elastic trusses leading to continua with exotic mechanical interactions. J. Phys. Conf. Ser. 319(1), 012018 (2011)

87. Solyaev, Y., Lurie, S., Barchiesi, E., et al.: On the dependence of standard and gradient elastic material constants on a field of defects. Math. Mech. Solids 25(1), 35-45 (2020)

88. Steigmann, D.J., dell'Isola, F.: Mechanical response of fabric sheets to three-dimensional bending, twisting, and stretching. Acta. Mech. Sin. 31, 373-382 (2015)

89. Steigmann, D.J., Pipkin, A.C.: Equilibrium of elastic nets. Philos. Trans. R. Soc. Lond. Ser. A Phys. Eng. Sci. 335(1639), 419-454 (1991)

90. Stochino, F., Cazzani, A., Poppi, S., et al.: Sardinia radio telescope finite element model updating by means of photogrammetric measurements. Math. Mech. Solids 22(4), 885-901 (2017)

91. Sumbatyan, M.A. (ed.): Wave Dynamics, Mechanics and Physics of Microstructured Metamaterials: Theoretical and Experimental Methods, vol. 109. Springer, Berlin (2019)

92. Temple, G.F.J., Bickley, W.G.: Rayleigh's Principle and Its Applications to Engineering: The Theory and Practice of the Energy Method for the Approximate Determination of Critical Loads and Speeds. Courier Corporation, North Chelmsford (2004)

93. Tomićević, Z., Hild, F., Kodvanj, J. et al.: Validation of elastic and identification of plastic parameters of Johnson-Cook law using DIC. In: 30th Danubia-Adria Symposium on Advances in Experimental Mechanics (2013) 
94. Toupin, R.: Theories of elasticity with couple-stress. Arch. Ration. Mech. Anal. 17(2), 85-112 (1964)

95. Turco, E., Barchiesi, E.: Equilibrium paths of Hencky pantographic beams in a three-point bending problem. Math. Mech. Complex Syst. 7(4), 287-310 (2019)

96. Turco, E., dell'Isola, F., Cazzani, A., et al.: Hencky-type discrete model for pantographic structures: numerical comparison with second gradient continuum models. Z. Angew. Math. Phys. 67, 85 (2016)

97. Turco, E., dell' Isola, F., Rizzi, N.L., et al.: Fiber rupture in sheared planar pantographic sheets: numerical and experimental evidence. Mech. Res. Commun. 76, 86-90 (2016)

98. Turco, E., Golaszewski, M., Cazzani, A., et al.: Large deformations induced in planar pantographic sheets by loads applied on fibers: experimental validation of a discrete Lagrangian model. Mech. Res. Commun. 76, 51-56 (2016)

99. Turco, E., Misra, A., Pawlikowski, M., et al.: Enhanced Piola-Hencky discrete models for pantographic sheets with pivots without deformation energy: numerics and experiments. Int. J. Solids Struct. 147, 94-109 (2018)

100. Wang, C.M., Zhang, H., Gao, R.P., et al.: Hencky bar-chain model for buckling and vibration of beams with elastic end restraints. Int. J. Struct. Stab. Dyn. 15(07), 1540007 (2015)

101. Yildizdag, M.E., Demirtas, M., Ergin, A.: Multipatch discontinuous Galerkin isogeometric analysis of composite laminates. Contin. Mech. Thermodyn. (2018). https://doi.org/10.1007/s00161-018-0696-9

102. Yildizdag, M.E., Tran, C.A., Barchiesi, E., et al.: A multi-disciplinary approach for mechanical metamaterial synthesis: a hierarchical modular multiscale cellular structure paradigm. In: Altenbach, H., Ochsner, A. (eds.) State of the Art and Future Trends in Material Modeling, pp. 485-505. Springer, Cham (2019)

Publisher's Note Springer Nature remains neutral with regard to jurisdictional claims in published maps and institutional affiliations. 